\title{
Application of Local Search for Optimal Assignment of Food Exchange Lists Problem
}

\author{
Achara Dholvitayakhun and Jukkrit Kluabwang, Member, IACSIT
}

\begin{abstract}
This paper proposed an efficient local search (LS) technique for solving optimal assignment of food exchange lists problem under these constraints, energy and quantity limits. The proposed LS is improved by backtracking mechanism (BT). The objective function and its constraints were explained and conducted how to map into a real value for LS to minimize subjected to constraints. Experimental tests were divided into three cases, underweight-male, normal weight-female and overweight-female. Computational efforts were set to 20 trials for each case. The results show that the proposed LS can confirm significantly reduction of searching time for each complete assignment of a food exchange list, many thousands times with respect to a man doing.
\end{abstract}

Index Terms-Food exchange list, ideal body weight, local search technique, backtracking mechanism.

\section{INTRODUCTION}

Nowaday both malnutrition and over nutrition conditions or obesity are being global problems, according to the World Health Organization (WHO) report in 2008 [1] found that more than 1.4 billion world population were overweight and $65 \%$ of world's population live in countries where overweight and obesity kills more people than underweight. To reduce the number of victim from the problem, it may be easily solved by energy balance method [2] and also food exchange list for each person is the core process of this method, according to food pyramid shown in Fig. 1.

In fact, the manual making assingment of food exchange list problem is tedious, time-consuming and uneconomical. In order to improve the efficiency and decrease the assigning time, an efficient heuristic search technique, namely local search [3] is introduced to solve the optimal assignment of food exchang list problem.

This paper consists of introduction, procedure of assigning food exchange list, proposed local search technique, objective function, computational results and summaries.

\section{Procedure OF Assigning FoOd ExChange List}

Steps by step of solving food exchange list mannually can be summarized as follows [4],

Manuscript received August 18, 2013; revised November 15, 2013. This work was support by Rajamangala University of Technology Lanna Tak, Thailand.

A. Dholvitayakhun is with Agricultural Industry Department, Rajamangala University of Technology Lanna, Tak 63000 Thailand (e-mail: achara2518@yahoo.co.th).

J. Kluabwang is with the Electrical Engineering Department, Rajamangala University of Technology Lanna, Tak 63000 Thailand (e-mail: jukkrit_k@rmutl.ac.th).
1) Calculate the ideal body weight (IBW) from each person's height in equation (1)

$I B W($ man $)=($ Heightincemtimeter -100$)$

or $I B W($ woman $)=($ Heightin cemtimeter -100$) \times 0.9$

2) Calculate energy required in a day from the obtained IBW.

3) Divide the obtained energy into three parts, protein: oils: carbohydrate, in percentage 20:20:60 respectively, for this study.

4) Assign food exchange list in portion according to the required energy into 6 food groups shown in Fig.1, fruits, vegetables, grains, protein foods, milk and oils.

5) Evaluate the strict constaints, error limit of energy be less than 30 kilocalories (kcal) a day, and each error limits of protein, oils and carbohydrate be less than 3 grams a day. If all constaints are met, then go forward to step 6) otherwise go back to step 4)

6) Stop assigning and reporting.

Practically, a man who have do a food exchange list often spends more and more time through these steps to finish each case since it is looked like a hard to solve problem. To reduce the tedious time, this paper proposes an efficient local search technique to solve this problem iteratively and successfully.

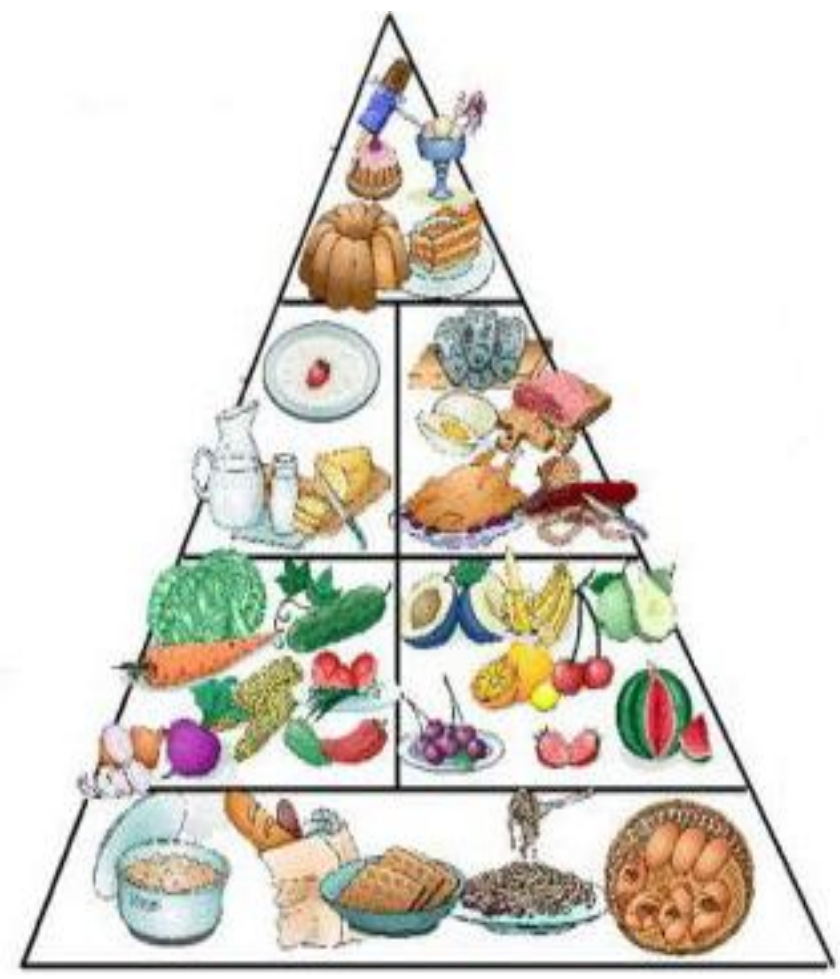

Fig. 1. Food pyramid. 


\section{Proposed Local Search Technique}

Heuristic search techniques play an important role in solving many difficult problems of both science and engineerig sides [5]-[7]. Local search (LS) is a basic heuristic search [3] and easy to understand. The procedure of the original local search can be summarized as follows,

1) Initialize parameters of LS.

2) Select an initial solution and evaluate its objective function.

3) Set the current solution as the best soluton.

4) Generate and evaluate neighborhood solutions and then choose the best one of neighborhood solution.

5) Update the best solution if the best neighborhood solution is better than the best solution otherwise keeping the same.

6) Stop searching if termination criterion (solution quality and time limit), namely TC, are met otherwise go back to step 4)

This paper has developed the original LS by adding an improved mechanism called backtracking mechanism (BT) after the step 5). The BT will be invoked when the best solution has been unupdated for a long time and then it will replace the current solution with the visited solution before because it may help unlocking from the trapped area to the new direction.

\section{OBJECTIVE Function AND PARAMETER SETTING}

From the steps of food exchange list problem described in the topic 2, the problem can formulated into minimization problem of optimization problem scheme as shown in equation (2)

$$
\begin{array}{ll}
\min & J(x) \\
\text { subjecto } & g(x)
\end{array}
$$

where $J(x)$ is an objective function, $x$ is input vector and $g(x)$ is constraint $(s)$. The $x$ input vector has 12 members, $x_{1}$ to $x_{12}$ represented portion of whole milk, reduced fat milk, fat free milk, vegetable, fruits, sugar, grains, meat a-d and oils or lipid respectively. This paper built the object function by using penalty technique integrated both objective function value and all constraints into only one variable, $J(x)$, shown in equation (3).

$$
J(x)=a \cdot x+b \cdot g_{1}(x)+c \cdot g_{2}(x)+d \cdot g_{3}(x)
$$

where $a$ - $d$ are weighting function values, $a=1, b=c=d=5$, $a x$ is error limit of energy less than $30 \mathrm{kcal} / \mathrm{day}, b g_{2}(x)$ to $d g_{4}(x)$ is error limits, of carbohydrate portions, protein portion and lipid portion, less than 3 grams/day/portion. The BT mechanism is set backward to the $5^{\text {th }}$ solution before. The first TC, the maximum iteration is 10,000 iterations and the second TC, F is less than or equal 25 for overweight case, less than or equal 30 for underweight case and less than or equal 31 for normal weight case.

\section{Computational Results}

The objective function included constraints and also the proposed LS technique have been coded in MATLAB ${ }^{\circledR}$. Testing is on laptop computer Intel ${ }^{\circledR}$ Pentium $^{\circledR}$ CPU B940 2GHz, RAM 4GByte, HDD 400GByte and Window 7 OS. To ensure the experimental results, 20 trials have been done and collected the particular informations in Table I to Table III for obtained final objective function values, iteration used and time spent in seconds, respectively.

TABLE I: FINAL OBJECTIVE FUNCTION VALUES FROM 20 TRIALS

\begin{tabular}{lllll}
\hline \hline Case Studdy & Min. & Mean & Max. & Std. Dev. \\
\hline Underweight & 10.2800 & 21.0840 & 27.7800 & 5.9368 \\
Normal & 18.3300 & 25.4560 & 30.8300 & 4.7480 \\
Overweight & 15.0000 & 21.6250 & 25.0000 & 3.4674 \\
\hline \hline
\end{tabular}

TABLE II: SEARCHING ITERATIONS FROM 20 TRIALS

\begin{tabular}{lllll}
\hline \hline Case Studdy & Min. & Mean & Max. & Std. Dev. \\
\hline Underweight & 168 & 2,978 & 9,192 & 2,848 \\
Normal & 74 & 2,105 & 8,781 & 2,206 \\
Overweight & 106 & 2,368 & 7,480 & 2,104 \\
\hline \hline
\end{tabular}

TABLE III: SEARCHING TIME IN SECOND FROM 20 TRIALS

\begin{tabular}{lllll}
\hline \hline Case Studdy & Min. & Mean & Max. & Std. Dev. \\
\hline Underweight & 0.1298 & 21.0840 & 27.7800 & 5.9368 \\
Normal & 18.3300 & 25.4560 & 30.8300 & 4.7480 \\
Overweight & 15.0000 & 21.6250 & 25.0000 & 3.4674 \\
\hline \hline
\end{tabular}

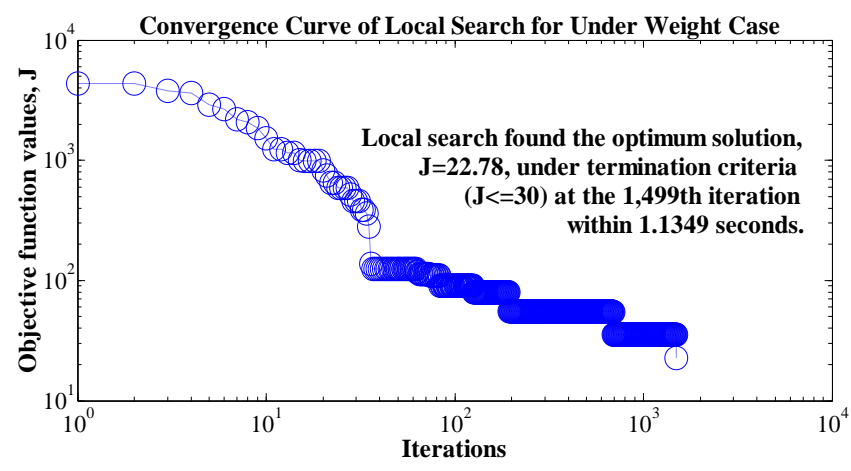

Fig. 2. Convergence curve.

The average final objective values illustrated in Table I show that the average quality of solution in the overweight case (21.6250) is better than of the normal case (25.456) and the underweight case is the best (21.0840). It can say that the proposed LS can find well for very good solution in overweight case.

Table II shows the number of iterations spent in each case. If we consider the average value in mean column, the normal case spent 2,105 iterations, underweight case spent 2,978 iterations and 2,368 iterations of the overweight case. Iterations is directly related to the searching time, if you spent more iteration it is easy to understand that you have to spent more time to search (take a look in Table III).

Table III demonstrates all case of searching time and the fastest trial occurred in overweight-male case where the proposed LS spent only 0.0708 seconds to reach the TC. If 
human doing the same job is approximated to spend 15 minutes, the higher speed of LS is more than 12,700 times with respect to of human doing.

Convergence property of LS on the problem of food exchange list can be illustrated by a curve in Fig. 2. It is of underweight case. Its track begins at $J=400$ and gradually goes down to the end at $J=22.78$ under one of termination criteria within 1.13 second of the $1,499^{\text {th }}$ iteration.

Fig. 3 demonstrates a sample of output report after the proposed LS met a TC of overweight-female case. Its obtained solution is a set of 12 portions, whole milk to lipids. After instead these portions into equation (2), it will return objective function value $J=22.5$ that is less than the specified $\mathrm{TC}=25$. The final solution is Whole milk 1 serving, reduced fat milk 1.5 serving, fat free milk 5 serving, vegetable b 7.5 serving, fruits 9 serving, sugar 1 serving, grains 0 serving, meat a 0 serving, meat b 1.5 serving, meat c 0.5 serving, meat d 0 serving and lipid 3 serving. Sum of obtained energy is $1,802.5 \mathrm{kcal}$ and differs from the required energy $2.5 \mathrm{kcal}$ which is acceptable in constraint error of energy no than 30 kcal.

\begin{tabular}{|c|c|c|}
\hline Sex & $:$ & Female \\
\hline Height in centimetre & $:$ & 167.0 \\
\hline Weight in kilograms & : & 75.0 \\
\hline Weight status & : & Over \\
\hline Whole milk & : & 1.0 \\
\hline Reduced fat milk & : & 1.5 \\
\hline Fat free milk & $:$ & 5.0 \\
\hline Vegetable b & $:$ & 7.5 \\
\hline Fruits & : & 9.0 \\
\hline sugar & : & 1.0 \\
\hline Grains & : & 0.0 \\
\hline Meat a & : & 0.0 \\
\hline Meat $b$ & : & 1.5 \\
\hline Meat c & $:$ & 0.5 \\
\hline Meat d & : & 0.0 \\
\hline Lipid & : & 3.0 \\
\hline Energy required in a day (kcal) & : & 1800.0 \\
\hline Carboh. required in grams & : & 270.0 \\
\hline Lipid required in grams & : & 40.0 \\
\hline Protien required in grams & : & 90.0 \\
\hline Energy assigned in a day (kcal) & : & 1802.5 \\
\hline Carboh. assigned in grams & : & 267.5 \\
\hline Lipid assigned in grams & : & 39.5 \\
\hline Protien assigned in grams & : & 89.0 \\
\hline Energy error in a day (kcal) & : & 2.5 \\
\hline Carboh. error in grams & : & $-2 \cdot 5$ \\
\hline Lipid error in grams & : & -0.5 \\
\hline Protien error in grams & : & -1.0 \\
\hline Calculation time in seconds & : & 8.14246055 \\
\hline
\end{tabular}

Fig. 3. Sample report of the proposed LS after finish searching.

\section{CONCLUSION}

This paper proposed an improved local search by backtracking mechanism for solving optimal assignment of food exchange list problem insteading classical human doing. The objective function is to be minimized and its constraints are error limit of energy less than $30 \mathrm{kcal} /$ day and error limits of three portions, carbohydrate, protein and lipid, less than 3 grams/day/portion. Experimental tests were divided into three cases, underweight-male, normalweight-female and overweight-female. Computational efforts were 20 trials for each case. As the results, the proposed LS can found significantly reduction of searching time for each complete assignment of a food exchange list, more than many thousands times with respect to human doing.

\section{ACKNOWLEDGMENT}

Authors would like to thank Control System Laboratory for MATLAB $^{\circledR}$ licensed support and staff for valuable advices.

\section{REFERENCES}

[1] World Health Organization Fact Sheets. Obesity Facts. [Online] Available: http://www.who.int/mediacenter/factsheets/fs311/en/

[2] M. Nelms, P. S. Kathryn, L. Karen, and L. R. Sara, Nutrition Therapy and Patholphysiology, 2nd ed. Belmont, CA: Wadsworth, 2011, ch. 12, pp. 238-282.

[3] V. Arya, N. Garg, R. Khandekar, A. Meyerson, K. Munagala, and V. Pandit, "Local search heuristics for k-median and facility location problems," SIAM J. on Computing, vol. 33, no. 3, pp. 544-562, 2004.

[4] L. K. Debruyne, K. Pinna, and E. N. Whitney, Nutrition and Therapy, 8th ed. Belmont, CA: Wadsworth, 2012, ch. 14, pp. 386-392.

[5] D. T. Pham and D. Karaboga, Intelligent Optimization Techniques: genetic algorithms, tabu search, simulated annealing and neural networks, London:Springer-Verlag, 2000, ch. 1, pp. 27-47.

[6] J. Kluabwang, D. Puangdownreong, and S. Sujitjorn, "Multipath adaptive tabu search for a vehicle control problem," Hindawi J. Appl. Math., vol. 2012, Article ID: 731623, 2012

[7] J. Kluabwang, "Modified adaptive tabu search algorithms for economic load dispatch," J. Indus. Tech., vol. 8, no. 2, pp. 59-67, May 2012 .

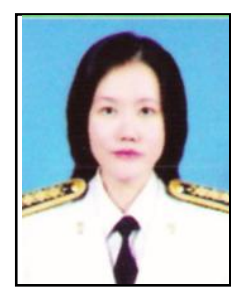

Achara Dholvitayakhun was born in Bangkok, Thailand in 1975. She received her M.E.H. degree in Food and Nutrition from Kasetsart University, Thailand in 2001 and Ph.D. in Food Technology degree from Mahasarakham University, Thailand in 2011. Currently she is working as a lecturer in the Faculty of Science and Agricultural Technology, Rajamangala University of Technolgoy Lanna Tak. Her current research interests include food and nutrition, diet therapy and food processing.

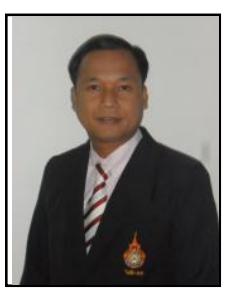

Jukkrit Kluabwang was born in Bangkok, Thailand in 1973. He received his B.Eng, M.Eng and Ph.D. in Electrical Engineering from Chiang Mai University, Chulalongkorn University and Suranaree University in 1995, 2004 and 2011, respectively. He has been with Electrical Engineering Department of Rajamangala University of Technology Lanna since 1997 as a lecturer. He is a member of IACSIT and also an IEEE member in Computational Intelligence Society. His current research is focusing on development and applications of heuristic search algorithms for solving electrical engineering problems and any problem in nutrition field. 